\title{
Durable Clinical Benefit in Patients with Advanced Cutaneous Melanoma after Discontinuation of Anti-PD-1 Therapies Due to Immune-Related Adverse Events
}

\author{
Umang Swami $\mathbb{D}^{1},{ }^{1}$ Varun Monga, ${ }^{2}$ Aaron D. Bossler, ${ }^{3}$ \\ Yousef Zakharia, ${ }^{2}$ and Mohammed Milhem $\left(\mathbb{1}^{2}\right.$ \\ ${ }^{1}$ Division of Oncology, Department of Internal Medicine, Huntsman Cancer Institute, University of Utah, Salt Lake City, UT, USA \\ ${ }^{2}$ Division of Hematology, Oncology and Blood \& Marrow Transplantation, University of Iowa Hospitals and Clinics, \\ 200 Hawkins Dr., Iowa City, IA 52242, USA \\ ${ }^{3}$ Department of Pathology, University of Iowa Hospitals and Clinics, 200 Hawkins Dr., Iowa City, IA 52242, USA
}

Correspondence should be addressed to Mohammed Milhem; mohammed-milhem@uiowa.edu

Received 4 March 2019; Revised 30 May 2019; Accepted 15 July 2019; Published 25 July 2019

Guest Editor: Shalini Gupta

Copyright (C) 2019 Umang Swami et al. This is an open access article distributed under the Creative Commons Attribution License, which permits unrestricted use, distribution, and reproduction in any medium, provided the original work is properly cited.

\begin{abstract}
Introduction. Anti-PD-1 therapies, pembrolizumab and nivolumab, are currently the standard of care for treatment of patients with metastatic melanoma. Treatment is usually continued until toxicity or disease progression. Though these therapies are well tolerated, some patients discontinue them due to immune-related adverse events (irAE). Discontinuation of therapy brings challenges to their management due to limited treatment options and lack of long-term prognostic information for these patients. Herein, we reviewed patients at our institution to analyze their clinical outcomes. Materials and Methods. Charts of 1264 consecutive patients enrolled between 8/1/2012 and 7/31/2017 at Melanoma Skin \& Ocular Tissue Repositories at Holden Comprehensive Cancer Center at the University of Iowa Hospitals and Clinic were reviewed. Eligible patients were those who received single-agent anti-PD1 therapy and subsequently discontinued it due to irAE. Reviewed data included patient demographics, prior medical history, baseline disease parameters, and outcomes. Kaplan-Meier survival analysis was done to determine progression-free survival (PFS) and overall survival (OS). Results. Overall 169 patients with advanced, unresectable, or metastatic cutaneous melanoma received anti-PD-1 therapy of which 16 (9.5\%) white, non-Hispanic patients with median age of 64.5 (range 35 to 81 years) discontinued treatment due to irAE. Fifteen patients received pembrolizumab and one received nivolumab. The median duration of treatment was 4.7 (range 0.7 to 11.5) months. Median follow-up was 30.3 (range 4.6 to 49.4) months. Median PFS was 24.6 months and median OS was not reached. Durable clinical benefit (time to progression or next treatment of more than 6 months from last treatment) was observed in 13 (81.2\%) patients. At the time of analysis, 8 patients had progressed and 4 patients died (all-cause). Discussion. Our results suggest that advanced melanoma patients discontinuing anti-PD-1 therapy due to irAE usually experience durable clinical benefit. However, caution is needed with these agents in patients with underlying autoimmune diseases.
\end{abstract}

\section{Introduction}

Monoclonal antibodies targeting programmed cell death 1 protein (PD-1) have shown to improve progression-free survival (PFS) and overall survival (OS) in patients with metastatic melanoma [1]. The advent of anti-PD-1 antibodies along with antibodies targeting cytotoxic T-lymphocyteassociated protein 4 (CTLA-4) and therapies targeting BRAF mutation has provided multiple options to treat patients with metastatic melanoma. Due to these therapies, the median overall survival of metastatic melanoma has improved from 6 months to more than 3 years [2-4]. Currently, two monoclonal antibodies targeted against PD-1 have been approved as first-line agents for the treatment of metastatic melanoma [1].

PD-1 inhibitors can lead to durable responses $[1,5]$ and have better toxicity profiles as compared to CTLA-4 inhibitors and targeted therapies $[1,3,4]$. However, approximately, $86 \%$ of patients experiencing treatment-related toxicities (all grades) and severe (grade 3 or higher) toxicities are in the range of 17 to $22 \%[3,4]$. Treatment discontinuation due to 
immune-related adverse events (irAEs) is estimated to occur in $15 \%$ to $25 \%$ of patients $[3,4]$. These patients lack effective therapies as many of them do not have actionable mutation, and even in patients with BRAF mutation, the median PFS with BRAF-MEK inhibitors is low (11 to 15 months) with a high rate of toxicities $[1,6,7]$. Therefore, there is a need to understand the long-term prognosis of patients who undergo treatment discontinuation due to irAE to guide management decisions.

\section{Materials and Methods}

After approval from the Institutional Review Board, data of 1264 patients enrolled at Melanoma Skin \& Ocular Tissue Repositories at Holden Comprehensive Cancer Center at the University of Iowa Hospitals and Clinics from 8/1/2012 to $7 / 31 / 2017$ was reviewed. Patients with unresectable, advanced, or metastatic cutaneous melanomas who discontinued antiPD-1 therapies due to irAEs were identified and their charts were reviewed in detail. Reviewed data included demographics (gender, race, and ethnicity), mutational status, prior treatment regimens including radiation therapy, melanoma metastases to brain and liver, and irAEs. Identified patients were followed till 02/26/2019. Progression (clinical or radiological) and responses were determined by iRECIST [8] and clinic notes. Outcomes with anti-PD-1 therapies including PFS, time from treatment discontinuation to progression, and OS were collected. Common Terminology Criteria for Adverse Events Criteria Version 4.03 were used to grade irAE [9].

2.1. Statistical Analysis. Baseline clinical and disease characteristics were summarized as medians and ranges for continuous variables and as numbers and percentages for categorical variables. Kaplan-Meier survival analysis was used to determine PFS and OS. Time was calculated from initiation of anti-PD-1 treatment to progression or, new treatment for PFS, time from last treatment to next treatment or progression for clinical benefit and to death due to any cause for OS. Durable clinical benefit was defined as time of 6 months or more to progression or next treatment from last therapy. Survival curves were drawn using GraphPad Prism Version 7.04 (GraphPad Software, Inc., San Diego, CA).

\section{Results}

3.1. Baseline Characteristics. Overall 169 patients with advanced, unresectable, or metastatic cutaneous melanoma received anti-PD-1 therapy, of which 16 (9.5\%) patients discontinued treatment due to irAEs. All patients who discontinued treatment were white and non-Hispanic. The median age was 64.5 (range 35 to 81 ) years. Ten $(62.5 \%)$ patients were male and six (37.5\%) were female. Eight (50\%) patients had a BRAF mutation. Four (25\%) patients had brain metastasis and one (6.3\%) had liver metastasis. Six (37.5\%) patients had prior treatment with ipilimumab for metastatic disease. Table 1 provides the baseline demographics and clinical profile of all patients.
3.2. Treatment. Fifteen (93.7\%) patients received pembrolizumab and one (6.3\%) patient received nivolumab. Eight (50\%) received anti-PD-1 therapy as the first line, seven $(43.7 \%)$ as second, and one $(7.3 \%)$ as the third line. Patient 6 received bevacizumab in addition to pembrolizumab after 6 cycles of single-agent pembrolizumab due to pseudoprogression in the brain. None of the patients received concomitant radiation with anti-PD-1 therapy. The median duration of treatment was 4.7 (range 0.7 to 11.5) months. Table 2 provides a summary of treatment duration, line of therapy, and outcomes of each patient.

3.3. irAEs. The median duration from initiation of treatment to development of irAE was 4 (range 0.5-11.5) months. Most commonly observed toxicities leading to treatment discontinuation included diarrhea and rash (4/16, 25\% each) and arthritis $(3 / 16,18.7 \%)$. Other observed toxicities included colitis (2), neuropathy (2), pancreatitis (2), fatigue, nausea, nephritis, adrenal insufficiency, hypothyroidism, low mood, mouth sores, hepatitis, uveitis, and myasthenia flare (1 each). Ten (62.5\%) patients experienced grade 3 or higher toxicities. Table 3 provides a summary of these toxicities and immunosuppressive agents used for their management. Apart from the mentioned toxicities, patient 1 developed grade 2 hypothyroidism on day 81 and patient 8 developed grade 2 pityriasis lichenoides after 2 cycles secondary to pembrolizumab. However, both did not lead to treatment discontinuation.

3.4. Outcomes. Median follow-up was 30.3 (range 4.6 to 49.4 ) months. Eight (50\%) had complete response, five $(31.2 \%)$ had partial response, two $(12.5 \%)$ had stable disease, and one $(6.3 \%)$ had progressive disease as best response to treatment. At the time of analysis, 8 patients had progressed and 4 patients experienced all-cause mortality, of which one death was unrelated to melanoma. Median PFS was 24.6 months and median OS was not reached due to durable disease control (Figures 1 and 2). Durable clinical benefit was observed in $13(81.2 \%)$ patients.

Patients number 4 and 16 had a PFS of less than 6 months PFS (Figure 3). Patient 4 had underlying thymoma and patient 16 had myasthenia gravis. With pembrolizumab, they experienced severe neuroimmune toxicity and flare-up of myasthenia gravis, respectively, which caused rapid clinical deterioration. All patients except one received steroids (oral, topical, or ophthalmic), while two received steroidsparing agents. Of the eight patients who progressed, three were retreated with pembrolizumab-containing regimen. Of these three patients, one developed pembrolizumab induced psoriasis, while the remaining two tolerated it without any significant side effects (Table 2).

\section{Discussion}

Prior studies have given conflicting evidence with regard to the association of PD-1-related irAEs with survival outcomes in melanoma patients. Freeman-Keller et al. reported OS 


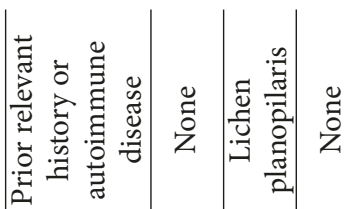

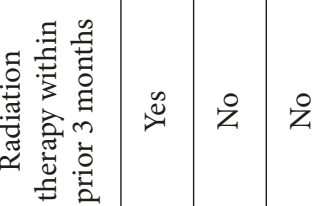

竞

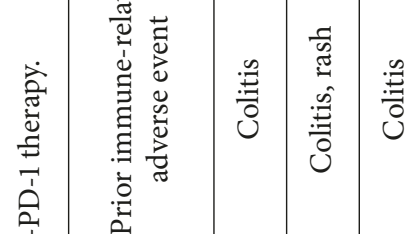

\section{西}

营

पै

.

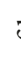

चुँ

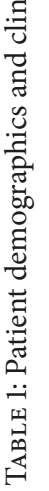

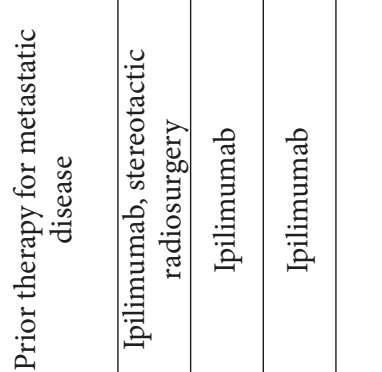

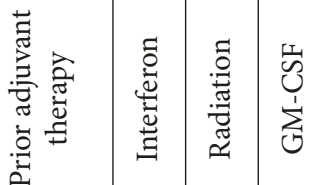

苞

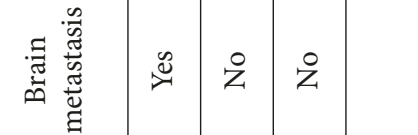

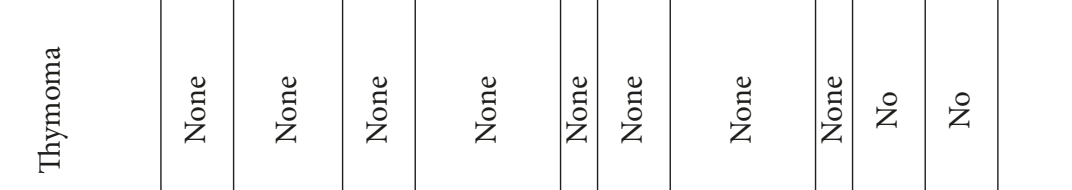

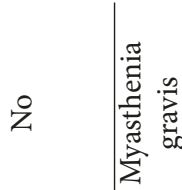

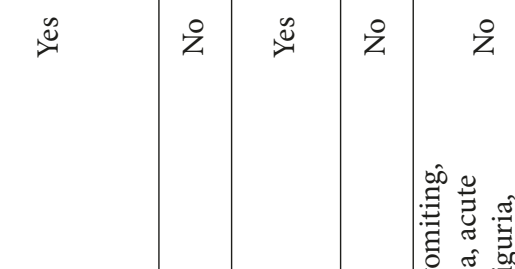

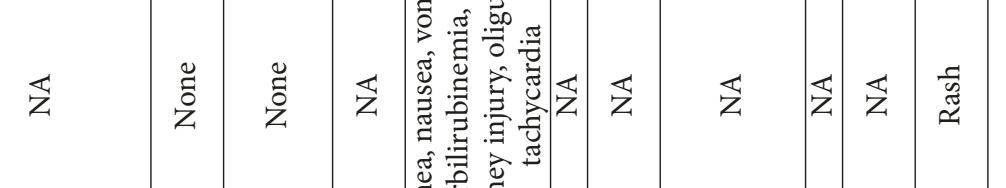

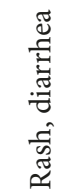

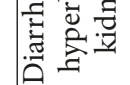

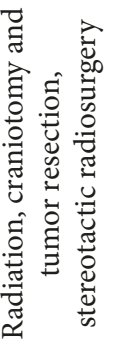

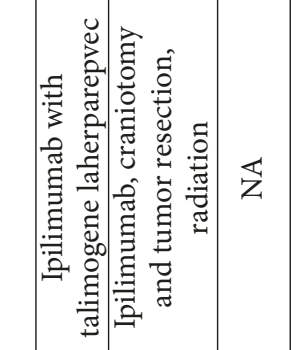

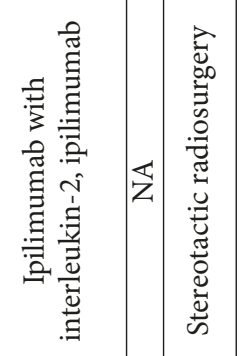

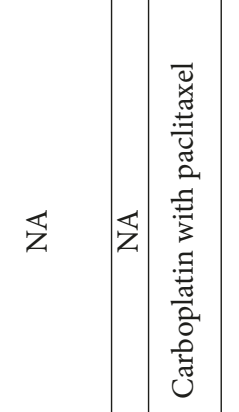

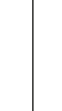

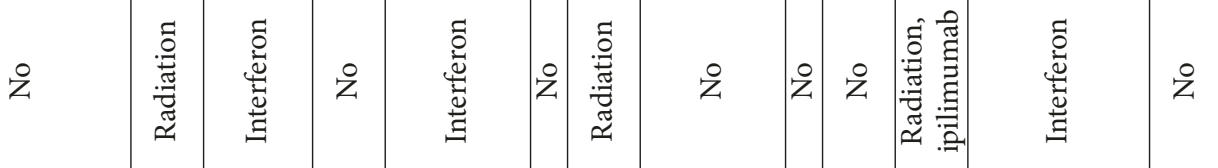

ż そ̊ z̊

z̊

z

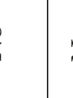

$\stackrel{2}{z} \stackrel{\circ}{z} \stackrel{\circ}{z}$

$\stackrel{2}{z} \stackrel{2}{z} \stackrel{2}{z}$

$\stackrel{Ð}{z}$

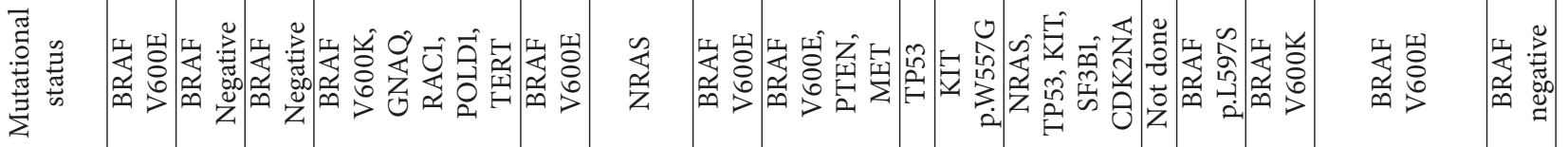

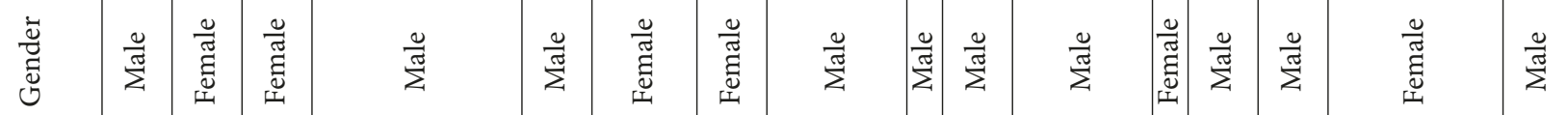

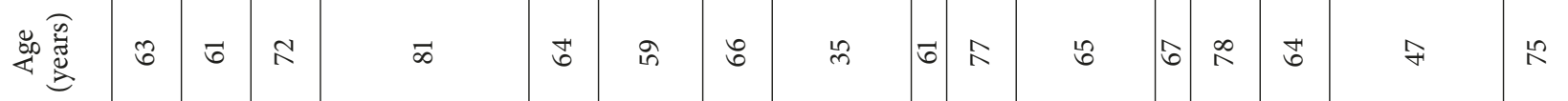

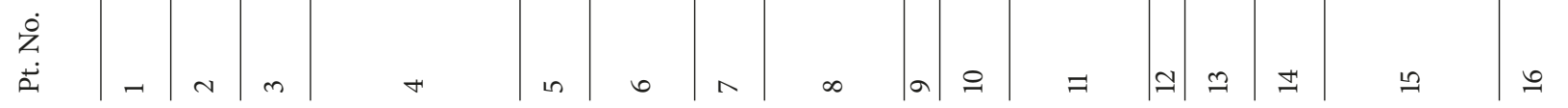




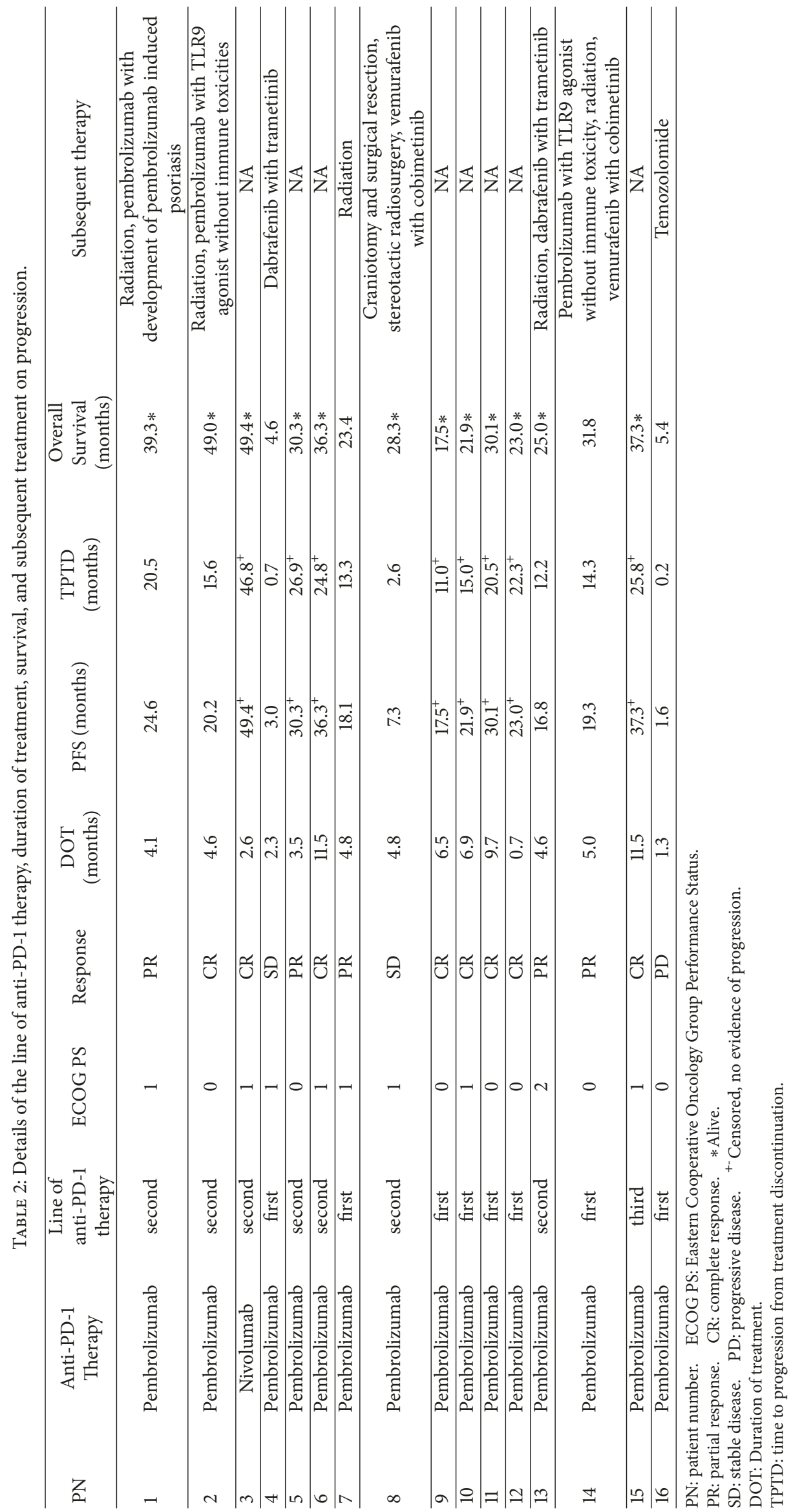


TABLE 3: Details of immune-related adverse events leading to discontinuation of anti-PD-1 therapy and their treatment.

\begin{tabular}{|c|c|c|c|}
\hline $\begin{array}{l}\text { Patient } \\
\text { Number }\end{array}$ & $\begin{array}{l}\text { irAE leading to discontinuation of } \\
\text { anti-PD-1 therapy }\end{array}$ & $\begin{array}{l}\text { Time of first presentation of any grade } \\
\text { irAE from initiation of therapy (months) }\end{array}$ & $\begin{array}{l}\text { Immune suppressive agents for } \\
\text { treatment of irAE }\end{array}$ \\
\hline 1 & $\begin{array}{c}\text { Grade } 2 \text { inflammatory arthritis and } \\
\text { neuropathy }\end{array}$ & 4.1 & Prednisone \\
\hline 2 & Grade 2 diarrhea & 4 & Budesonide \\
\hline \multirow[b]{2}{*}{3} & Grade 3 colitis and diarrhea & 2.6 & Prednisone, budesonide \\
\hline & Grade 2 adrenal insufficiency & 4.1 & Hydrocortisone \\
\hline 4 & Grade 3 sensorimotor polyneuropathy & 2.3 & Prednisone \\
\hline \multirow[b]{2}{*}{5} & Grade 1 diarrhea & 2.8 & Budesonide \\
\hline & Grade 3 pancreatitis and colitis & 3.5 & Budesonide \\
\hline 6 & Grade 3 rash & 11.5 & Dexamethasone, topical steroids \\
\hline 7 & Grade 2 inflammatory arthritis & 4.6 & Prednisone, methotrexate \\
\hline 8 & $\begin{array}{c}\text { Grade } 2 \text { fatigue, nausea, diarrhea, } \\
\text { arthritis }\end{array}$ & 4.8 & None \\
\hline 9 & Grade 3 pancreatitis & 6.5 & Prednisone \\
\hline 10 & Grade 2 nephritis & 6.9 & Prednisone \\
\hline 11 & $\begin{array}{l}\text { Grade } 2 \text { hypothyroidism, low mood, } \\
\text { mouth sores, rash }\end{array}$ & 9.7 & Topical steroids \\
\hline 12 & Grade 3 rash & 0.5 & Prednisone \\
\hline 13 & Grade 3 hepatitis & 2.1 & Prednisone, budesonide \\
\hline 14 & Grade 3 uveitis & 3.9 & $\begin{array}{l}\text { Prednisone, ophthalmic } \\
\text { prednisolone }\end{array}$ \\
\hline 15 & Grade 3 rash & 11.3 & Prednisone, topical steroids \\
\hline 16 & Grade 4 myasthenia flare & 0.8 & $\begin{array}{l}\text { Plasma exchange, mycophenolate } \\
\text { mofetil, prednisone, intravenous } \\
\text { immunoglobulin, abatacept }\end{array}$ \\
\hline
\end{tabular}

IrAE: immune-related adverse events.

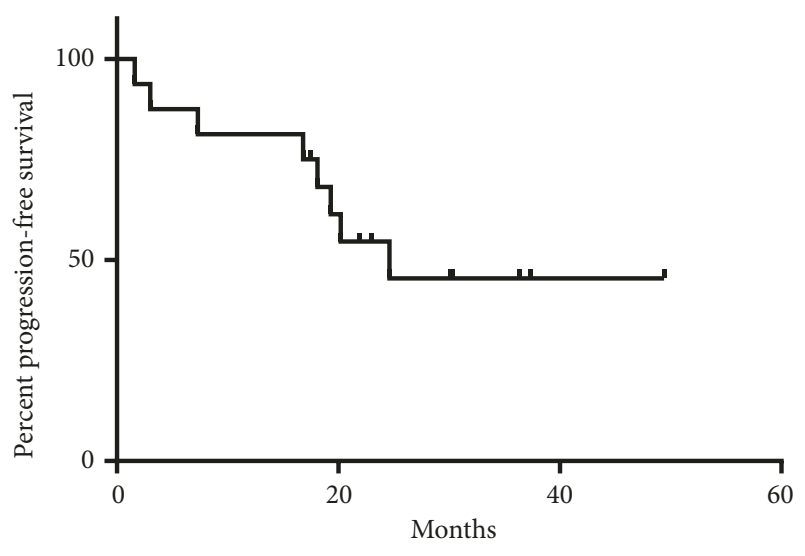

FIgURE 1: Kaplan-Meier curve for progression-free survival.

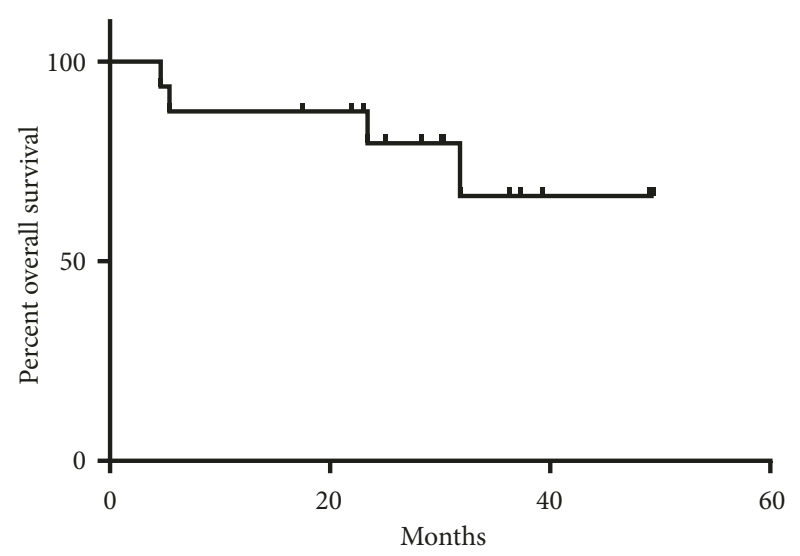

FIGURE 2: Kaplan-Meier curve for overall survival. association with rash and vitiligo, while no survival benefit was seen with other irAEs including endocrinopathies, colitis, or pneumonitis. In the study, 12-week landmark PFS analysis was difficult to interpret due to exclusion of a large number of patients [10]. Another pooled analysis of 576 patients by Weber et al. reported that after exclusion of patients progressing before 12 weeks, there was no difference in PFS between patients without AEs and those with one to two AEs or between those with any-grade AE and all patients [11]. In another study by Indini et al., irAE was associated with improved PFS [HR 0.47 (95\% CI 0.26, 0.86); $\mathrm{p}=0.016$ ] and OS [HR 0.39 (95\% CI 0.18, 0.81); p = 0.007] on multivariable analysis in patients who received more than 2 doses of antiPD-1 therapies [12]. Quach et al. reviewed single institution data of 318 patients treated with anti-PD-1 therapies with or without ipilimumab and reported a better response rate 


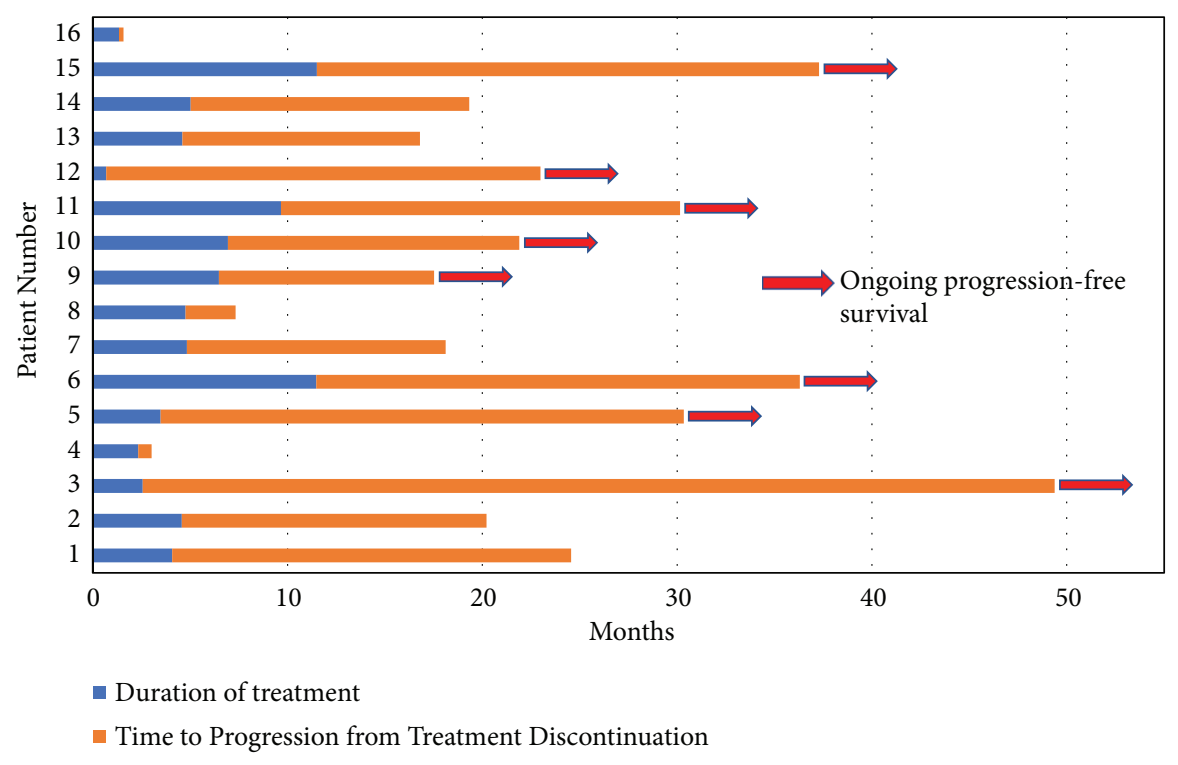

FIGURE 3: Swimmer's plot demonstrating duration of treatment and progression-free survival.

(60.0\% vs. $28.6 \% ; \chi^{2} p<.001$ ), median PFS (797 vs. 112 days; log rank $\mathrm{p}<.001$ ), and median OS (1691 vs. 526 days; $\mathrm{p}<.001)$ in patients who experienced cutaneous side effects. Superior outcomes with regard to response rate, PFS, and OS were seen with vitiligo and rash as compared to pruritus [13].

In this study, we analyzed the impact on PFS and OS for patients who discontinued anti-PD-1 therapy due to irAEs. Complete response was seen in patients with irAEs from a wide spectrum and grade of toxicities including grade 2 and 3 diarrhea/colitis ( 2 patients), grade 3 rash (3 patients), grade 3 pancreatitis ( 1 patient), grade 2 nephritis ( 1 patient), and grade 2 hypothyroidism, low mood, mouth sores, and rash (1 patient). We found a durable clinical benefit in thirteen patients (81.2\%) discontinuing PD-1 directed therapy due to irAEs; none of them progressed in more than one year and 6 $(37.5 \%)$ in more than 2 years (Figure 3 ). The benefit was not seen in patients with an underlying autoimmune disease like myasthenia gravis or who are at a higher risk of developing it, like patient 4 with thymoma [14]. However, because of the small sample size, no definitive conclusion should be drawn; this finding needs to be explored further.

KEYNOTE-001 has reported durable complete remission after discontinuation of pembrolizumab in patients with complete remission [5]. However, we found durable benefit in patients with residual disease also. Though the mechanisms for this phenomenon are not clear, it is very much possible that $\mathrm{PD}-1$ blockade may result in an adaptive memory immune response providing antitumor effect even after treatment cessation and translating as irAEs [15-17]. A similar study evaluated 19 patients with metastatic renal cell carcinoma who experienced an initial clinical response but after irAE discontinued PD-1/PD-L1 therapy. The median time on PD-1/PD-L1 therapy was 5.5 months; median TTP was 18.4 months and durable clinical benefit off treatment (TTP > 6 months) was observed in $68.4 \%(n=13)$ patients [15].
Pollack et al. reported that metastatic melanoma patients who discontinued CTLA4/PD-1 blockade due to irAEs can be rechallenged with anti-PD-1 therapies. The study showed a relatively higher rate of recurrent or different irAEs on resumption of anti-PD-1 therapies. They concluded that this approach can be used in selected patients [18]. We also found that patients who had to discontinue pembrolizumab due to irAE were able to be treated again with pembrolizumabbased therapies with manageable toxicities in two of the three patients. Our study has similar limitations as most retrospective studies including selection bias, chances of errors during data entry and confounding.

In summary, we present outcomes of 16 patients with metastatic melanoma who discontinued anti-PD-1 therapies due to immune toxicities. To the best of our knowledge, it is the largest series to date of real-world patients. Its strength includes a long-term follow-up and comprehensive analysis of each case which can help generate multiple hypotheses in combination with other relevant studies. Our results show durable clinical benefit in patients who discontinue anti-PD-1 therapies after irAEs. However, this needs to be confirmed in larger cohorts. We also need more comprehensive preclinical and clinical studies to determine how individual patient variables, cancer and immune system, interact to cause irAEs in only a select few patients while sparing a majority. We also need to develop predictive and prognostic novel biomarkers for anti-PD-1 therapies and also for irAEs.
Abbreviations
PD-1: Programmed cell death 1 protein
PFS: $\quad$ Progression-free survival
OS: Overall survival
CTLA-4: Cytotoxic T-lymphocyte-associated protein 4
irAE: Immune-related adverse events
TTP: Time to progression. 


\section{Data Availability}

The data used to support the findings of this study are available from the corresponding author upon request.

\section{Disclosure}

The authors received no specific funding for this work.

\section{Conflicts of Interest}

None of the authors have direct competing interest to the study. Umang Swami does not have any conflicts of interest. Varun Monga has research support from Immunocellular, Orbus Therapeutics, Deciphera, and NewLink Genetics. Aaron D. Bossler has received honorarium from Novartis and Roche. He is on advisory board of Novartis and Roche and has travel support from Novartis and Roche. Yousef Zakharia has institutional research support from NewLink. He is on the advisory board of Novartis, Pfizer, Roche Diagnostics, EISAI, Exelixis, JNJ, Castle Bioscience, and Amgen. Mohammed Milhem is on the advisory board of Genentech, BMS, EISAI, Novartis, EMD Serono, and Blueprint Medicines Corporation.

\section{Acknowledgments}

(1) HCCC Scientific Acknowledgment: "Research reported in this publication was supported by the National Cancer Institute of the National Institutes of Health under Award Number P30CA086862. The content is solely the responsibility of the authors and does not necessarily represent the official views of the National Institutes of Health." (2) MaST Scientific Acknowledgment: "Banked serum samples \& clinical data were obtained through the University of Iowa Melanoma, Skin \& Ocular Repositories (MaST), an Institutional Review Board-approved biospecimen repository."

\section{References}

[1] C. Luther, U. Swami, J. Zhang, M. Milhem, and Y. Zakharia, "Advanced stage melanoma therapies: Detailing the present and exploring the future," Critical Review in Oncology/Hematology, vol. 133, pp. 99-111, 2019.

[2] E. L. Korn, P. Liu, S. J. Lee et al., "Meta-Analysis of Phase II Cooperative Group Trials in Metastatic Stage IV Melanoma to Determine Progression-Free and Overall Survival Benchmarks for Future Phase II Trials," Journal of Clinical Oncology, vol. 26, no. 4, pp. 527-534, 2008.

[3] O. Hamid, C. Robert, A. Daud et al., "Five-year survival outcomes for patients with advanced melanoma treated with pembrolizumab in keynote-001," Annals of Oncology, 2019.

[4] F. S. Hodi, V. Chiarion-Sileni, R. Gonzalez et al., "Nivolumab plus ipilimumab or nivolumab alone versus ipilimumab alone in advanced melanoma (CheckMate 067): 4-year outcomes of a multicentre, randomised, phase 3 trial," The Lancet Oncology, vol. 19, no. 11, pp. 1480-1492, 2018.

[5] C. Robert, A. Ribas, O. Hamid et al., "Durable complete response after discontinuation of pembrolizumab in patients with metastatic melanoma," Journal of Clinical Oncology, vol. 36, no. 17, pp. 1668-1674, 2018.

[6] N. Grogan, U. Swami, A. D. Bossler, Y. Zakharia, and M. Milhem, "Toxicities with targeted therapies after immunotherapy in metastatic melanoma," Melanoma Research, vol. 28, no. 6, pp. 600-604, 2018.

[7] K. R. Saab, M. J. Mooradian, D. Y. Wang et al., "Tolerance and efficacy of BRAF plus MEK inhibition in patients with melanoma who previously have received programmed cell death protein 1-based therapy," Cancer, vol. 125, no. 6, pp. 884891, 2019.

[8] L. Seymour, J. Bogaerts, A. Perrone et al., "iRECIST: guidelines for response criteria for use in trials testing immunotherapeutics," The Lancet Oncology, vol. 18, no. 3, pp. e143-e152, 2017.

[9] US Department of Health and Human Services NIoH National Cancer Institute, Common terminology criteria for adverse events $v 4.03$ (ctcae), 2010, https://www.eortc.be/services/ doc/ctc/CTCAE_4.03_2010-06-14_QuickReference_5x7.pdf.

[10] M. Freeman-Keller, Y. Kim, H. Cronin, A. Richards, G. Gibney, and J. S. Weber, "Nivolumab in resected and unresectable metastatic melanoma: Characteristics of immunerelated adverse events and association with outcomes," Clinical Cancer Research, vol. 22, no. 4, pp. 886-894, 2016.

[11] J. S. Weber, F. S. Hodi, J. D. Wolchok et al., "Safety profile of nivolumab monotherapy: A pooled analysis of patients with advanced melanoma," Journal of Clinical Oncology, vol. 35, no. 7, pp. 785-792, 2017.

[12] A. Indini, L. Di Guardo, C. Cimminiello et al., "Immunerelated adverse events correlate with improved survival in patients undergoing anti-PD1 immunotherapy for metastatic melanoma," Journal of Cancer Research and Clinical Oncology, vol. 145, no. 2, pp. 511-521, 2019.

[13] H. T. Quach, A. K. Dewan, E. J. Davis et al., "Association of Anti-Programmed Cell Death 1 Cutaneous Toxic Effects With Outcomes in Patients With Advanced Melanoma," JAMA Oncology, vol. 5, no. 6, p. 906, 2019.

[14] C. Bernard, H. Frih, F. Pasquet et al., "Thymoma associated with autoimmune diseases: 85 cases and literature review," Autoimmunity Reviews, vol. 15, no. 1, pp. 82-92, 2016.

[15] D. J. Martini, L. Hamieh, R. R. McKay et al., "Durable Clinical Benefit in Metastatic Renal Cell Carcinoma Patients Who Discontinue PD-1/PD-L1 Therapy for Immune-Related Adverse Events," Cancer Immunology Research, vol. 6, no. 4, pp. 402-408, 2018.

[16] P. S. Kim and R. Ahmed, "Features of responding T cells in cancer and chronic infection," Current Opinion in Immunology, vol. 22, no. 2, pp. 223-230, 2010.

[17] G. P. Dunn, L. J. Old, and R. D. Schreiber, "The three Es of cancer immunoediting," Annual Review of Immunology, vol. 22, pp. 329-360, 2004.

[18] M. H. Pollack, A. Betof, H. Dearden et al., "Safety of resuming anti-PD-1 in patients with immune-related adverse events (irAEs) during combined anti-CTLA-4 and anti-PD1 in metastatic melanoma," Annals of Oncology, vol. 29, no. 1, pp. 250-255, 2018. 


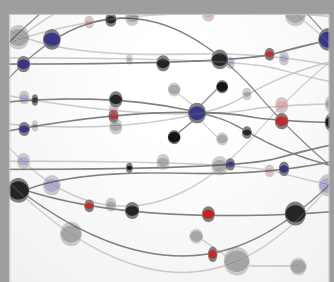

The Scientific World Journal
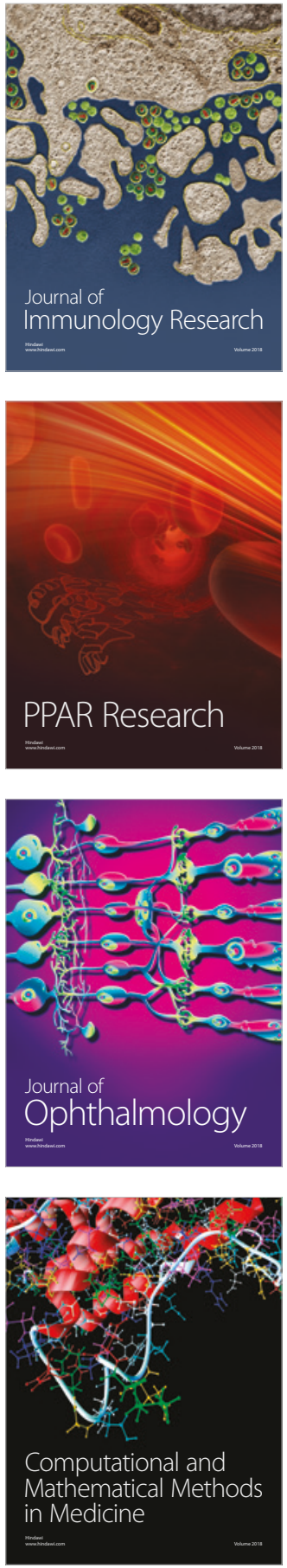

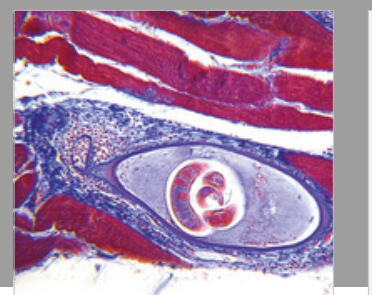

Gastroenterology Research and Practice

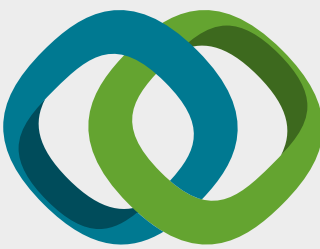

\section{Hindawi}

Submit your manuscripts at

www.hindawi.com
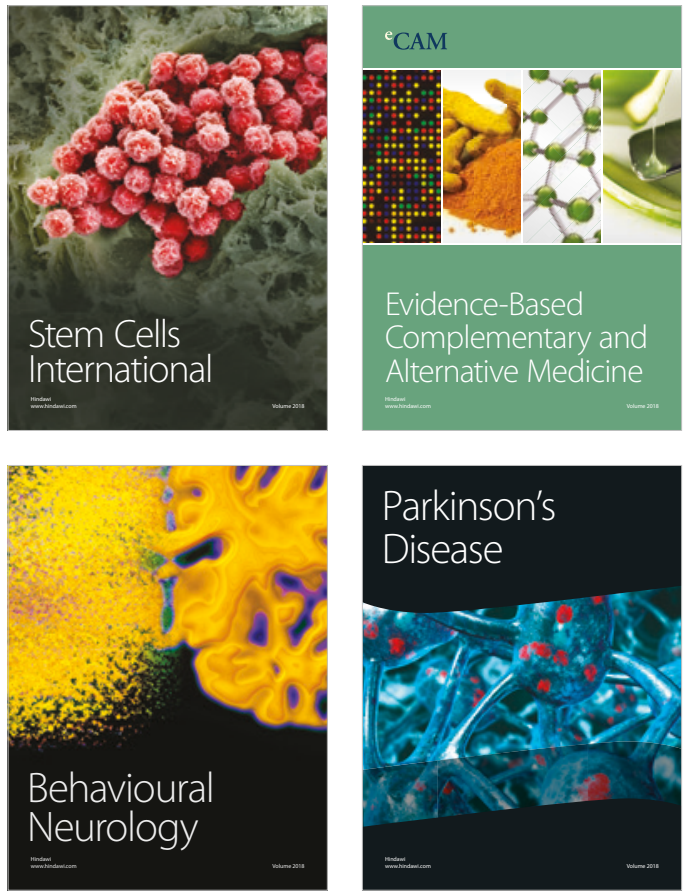

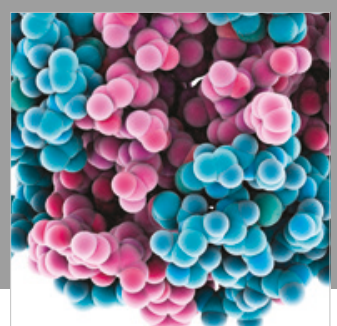

ournal of

Diabetes Research

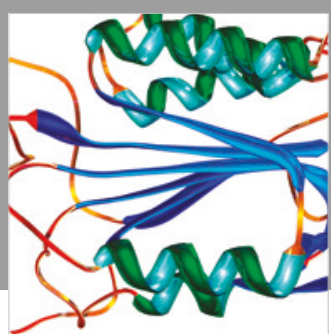

Disease Markers
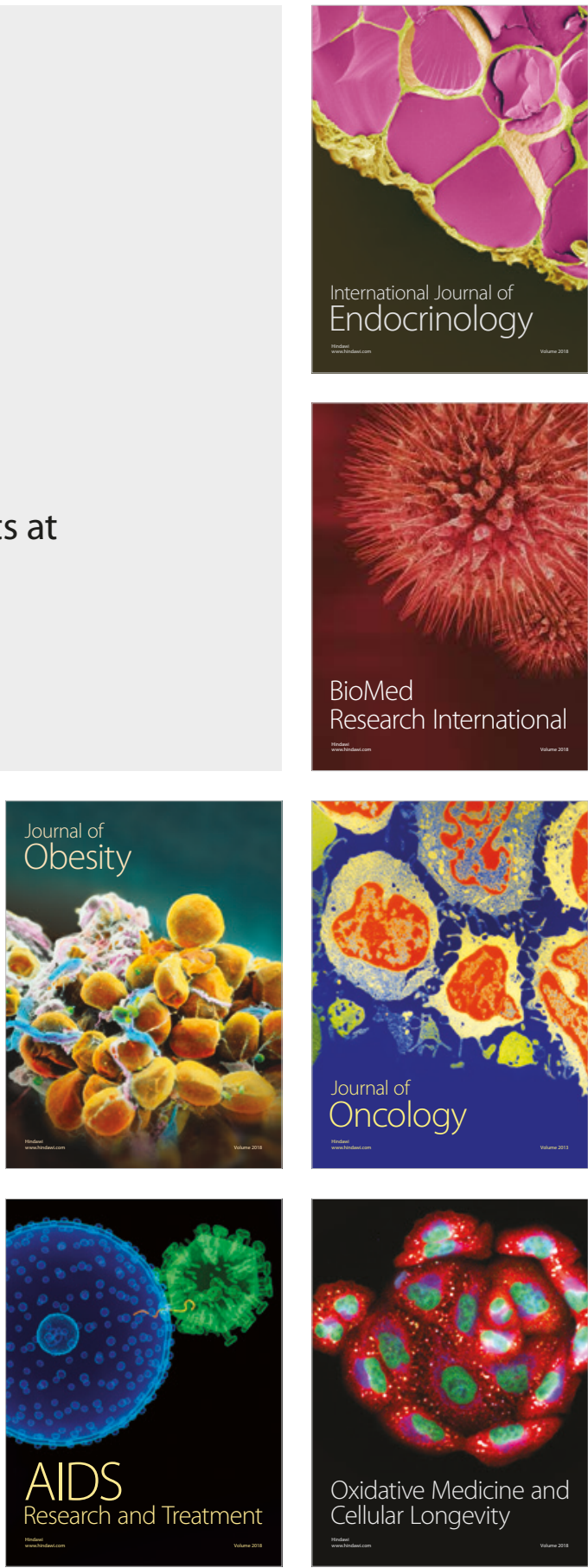